\section{IMPROVING THE NATION'S SMILE}

Oral-B are proud to support National Smile Month, the campaign to promote better dental health and awareness in the UK, which is now in its 36th year.

With the aid of Oral-B's sponsorship, the British Dental Health Foundation (BDHF) have conducted a revealing survey which highlights attitudes and trends toward dental health in the current climate. Despite economic obstacles, a majority of people still attach great importance to their smile and their dental health, opting by a substantial margin to both improve the appearance of their teeth and to maintain what they have.

The true footmen of National Smile Month are dental professionals themselves. Their tireless diligence in educating their patients about dental health issues under the National

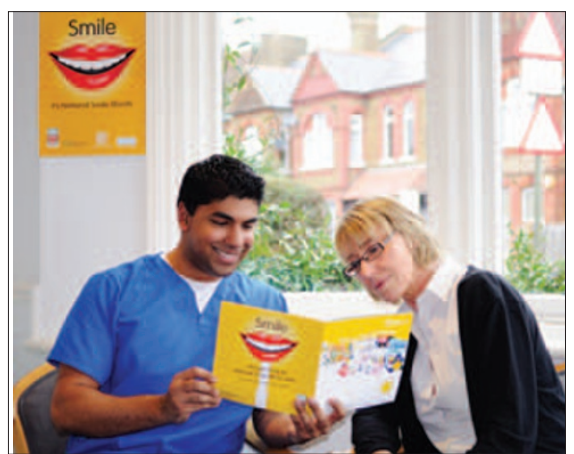

Smile Month umbrella has enabled the campaign to make a measurable difference in improving the state of the nation's smile. Dental professionals at all levels are encouraged once again to continue this valuable effort.

National Smile Month takes place from 20 May - 20 June 2012. Visit www.nationalsmilemonth.org for more information and resources.

\title{
QUANTIFY YOUR PATIENTS' TOOTH GRINDING
}

GrindCare Measure is a tool for accurately measuring whether your patients grind their teeth, and how often. Within just 4-5 days of use, GrindCare Measure identifies and quantifies the patient's grinding pattern.

GrindCare Measure monitors electromyographic (EMG) signals generated in the temporalis muscle. GrindCare Measure can be used for quantification of EMG activity during sleep and reduction of the EMG activity by applying electrical stimulation to the skin.

When a patient grinds their teeth, the GrindCare Measure electrode records the EMG signals and sends them to the GrindCare Measure device for subsequent analysis. GrindCare Measure records the number of bruxism episodes and bursts, the average duration of bursts and the total burst intensity.

The GrindCare Stimulator includes an amplifier, a microprocessor and a stimulus pulse generator and is as such a complete standalone biofeedback device. The stimulator has a built-in algorithm for monitoring and analysing EMG signals from the anterior muscle temporalis and detecting events of muscular activity based on these signals. When bruxing activity is detected, biofeedback is given in the form of mild electrical stimulation applied to the skin. The stimulation induces relaxation of the masseter and temporalis muscles without disturbing sleep. The delivery of this mild electrical stimulation to relax the muscle in turn inhibits the bruxing episode.

The GrindCare Measure can be loaned to the patient for home use. In less than a week, the device generates the data needed. The data can then be analysed and a diagnosis and treatment plan prepared.

Visit www.prestige-dental.co.uk.

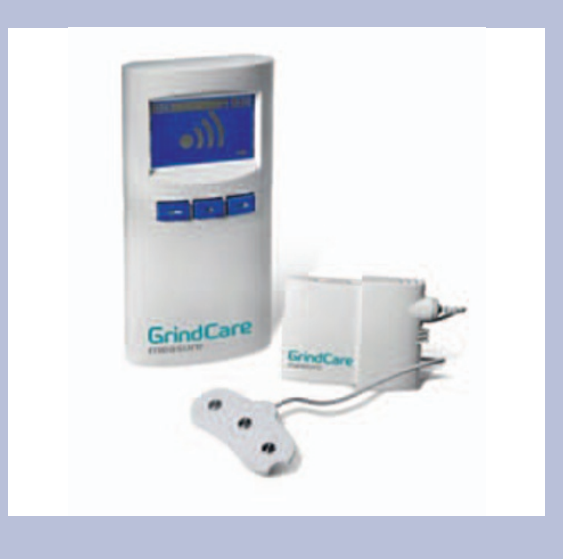

